\title{
Exogenous Application of Polycationic Nanobactericide on Tomato Plants Reduces the Candidatus Liberibacter Solanacearum Infection
}

\author{
Adela Nazareth García-Sánchez ${ }^{1}$, Roberto Yáñez-Macias ${ }^{1}{ }^{\mathbb{D}}$, José Luis Hernández-Flores ${ }^{2}$ (D), \\ Ariel Álvarez-Morales ${ }^{2}$, José Humberto Valenzuela-Soto ${ }^{1,3, * \mathbb{D}}$, Carlos Guerrero-Sanchez ${ }^{4,5}$ and \\ Ramiro Guerrero-Santos 1,*(D)
}

1 Centro de Investigación en Química Aplicada, Boulevard Enrique Reyna No. 140, Saltillo 25294, Mexico; nazareth_gs@hotmail.com (A.N.G.-S.); roberto.yanez@ciqa.edu.mx (R.Y.-M.)

2 Centro de Investigación y de Estudios Avanzados-Unidad Irapuato, Irapuato 36821, Mexico; jose.hernandezf@cinvestav.mx (J.L.H.-F.); ariel.alvarez@cinvestav.mx (A.Á.-M.)

3 CONACyT-Centro de Investigación en Química Aplicada, Boulevard Enrique Reyna Hermosillo 140, Saltillo 25294, Mexico

4 Laboratory of Organic and Macromolecular Chemistry (IOMC), Friedrich Schiller University Jena, Humboldtstr. 10, 07743 Jena, Germany; carlos.guerrero.sanchez@uni-jena.de

check for updates

Citation: García-Sánchez, A.N.; Yáñez-Macias, R.; Hernández-Flores, J.L.; Álvarez-Morales, A.;

Valenzuela-Soto, J.H.; Guerrero-Sanchez, C.; Guerrero-Santos, R. Exogenous Application of Polycationic Nanobactericide on Tomato Plants Reduces the Candidatus Liberibacter Solanacearum Infection. Plants 2021, 10, 2096. https://doi.org/10.3390/ plants10102096

Academic Editors: Michelina Ruocco and Maurilia Maria Monti

Received: 12 September 2021 Accepted: 28 September 2021 Published: 3 October 2021

Publisher's Note: MDPI stays neutral with regard to jurisdictional claims in published maps and institutional affiliations.

Copyright: (c) 2021 by the authors. Licensee MDPI, Basel, Switzerland. This article is an open access article distributed under the terms and conditions of the Creative Commons Attribution (CC BY) license (https:// creativecommons.org/licenses/by/ $4.0 /)$.
5 Jena Center for Soft Matter (JCSM), Friedrich Schiller University Jena, Philosophenweg 7, 07743 Jena, Germany

* Correspondence: humberto.valenzuela@ciqa.edu.mx (J.H.V.-S.); ramiro.guerrero@ciqa.edu.mx (R.G.-S.)

Abstract: Candidatus Liberibacter solanacearum ( $\mathrm{C} a \mathrm{Lso})$ is associated with diseases in tomato crops and transmitted by the tomato psyllid Bactericera cockerelli. A polymeric water-dispersible nanobactericide (PNB) was evaluated against $\mathrm{Ca}$ Lso as a different alternative. PNB is a well-defined polycationic diblock copolymer designed to permeate into the vascular system of plants. Its assessment under greenhouse conditions was carried out with tomato plants previously infected with CaLso. Using a concentration as low as $1.0 \mathrm{mg} \mathrm{L}^{-1}$, a small but significant reduction in the bacterial load was observed by real-time qPCR. Thus, to achieve an ecologically friendly dosage and set an optimum treatment protocol, we performed experiments to determine the effective concentration of PNB to reduce $\sim 65 \%$ of the initial bacterial load. In a first bioassay, a 40 - or 70 -fold increase was used to reach that objective. At this concentration level, other bioassays were explored to determine the effect as a function of time. Surprisingly, a real reduction in the symptoms was observed after three weeks, and there was a significant decrease in the bacterial load level ( 98\%) compared to the untreated control plants. During this period, flowering and formation of tomato fruits were observed in plants treated with PNB.

Keywords: nanobactericide; polycationic diblock copolymer; tomato plants; Candidatus Liberibacter solanacearum; Bactericera cockerelli

\section{Introduction}

Tomato (Solanum lycopersicum L.) is a herbaceous plant of the genus Solanum and the Solanaceae family, with more than 3000 species, including many plants of agricultural importance [1]. Tomato production is of the utmost economic relevance. Its production increases proportionally with the population growth. According to FAO, globally, the average tomato consumption reached $20.1 \mathrm{~kg}$ per capita in 2013 from an all-time low of $7.77 \mathrm{~kg}$ registered in 1963. However, this crop stands as one of the riskiest for growers. Many factors, such as the selection of cultivars, management practices, bio-aggressor attacks, and diseases, have generally threatened its production [2]. Indeed, it is known that there are more than 60 pathogens, including fungi, viruses, nematodes, and bacteria, which cause diseases and consequently generate significant economic losses all around 
the globe [3]. Such conditions have a higher incidence and impact when growers operate in open fields. That is why they gradually migrated towards the adoption of protected agriculture techniques [4]. Therefore, phytophagous insects and pathogen infections are severe, mainly when "good practices" are not rigorously followed [5].

The most relevant case is related to Bactericera cockerelli. This insect belongs to the Hemiptera order and the Triozidae family. It is considered one of the most serious pests for tomatoes, potatoes (Solanum tuberosum), peppers (Capsicum annum), and other nonnightshade crops. Bactericera cockerelli is recognized as the vector of Candidatus Liberibacter solanacearum ( $\mathrm{CaLso}$ ), a Gram-negative $\alpha$-proteobacteria lacking cell wall, and the causative agent of the "permanent" disease in tomato and "purple tip" or "zebra chip" in potato [6]. The infection process follows a consistent pattern, with a propagative, circulatory, and persistent transmission mode into the phloem, during insect feeding [7]. By staying in this tissue and consuming its nutrients, bacteria produce disease symptoms very similar to those that manifest when there is water or nutritional deficiency, such as growth retardation, internodes, and shortened petioles; necrosis in apical buds; and chlorosis, ascending curvatures of leaves, and abortion of flowers [8]. Due to its oligophagous feeding practice, $B$. cockerelli has a long history of exposure to many insecticides and repellents [9]; therefore, it has developed high resistance levels. Biological control, a green alternative to chemical pesticides, has also been proposed to fight these diseases; for instance, the effect of entomopathogenic fungi was recently reported [10,11], and various natural enemies of $B$. cockerelli adults and nymphs have been considered [12-14]. However, there are still no clear results in this regard, and, in general, additional research is necessary before authoritative practical guidance can be provided to resolve or lessen the problem.

On the other hand, the direct control of the pathogenic microorganism in the phloem is exceptionally challenging due to the slow migration of bactericidal agents within this complex tissue. The latter is probably due to the structural characteristics of the bactericidal molecules (e.g., water solubility) and the intricate internal vascular surface composition. Consequently, researchers have not usually undertaken a direct attack approach to CaLso [15-17]. Despite this, several pesticides have been tested unsuccessfully to control the disease produced by phloem-housed bacteria such as CaLso [18]. It is worth mentioning that the use of any bactericide, insecticide, or repellent is still far from being useful and sustainable. Moreover, the situation worsens due to the increased resistance of bacteria against different treatments, which increases the required amounts of bactericide substances, as well as costs, and environmental risks [19-21]

To skirt this issue, a nanotechnological approach is proposed in this contribution. Hence, a new low-dose polymeric nanobactericide (PNB) was thoroughly evaluated. PNB was recently developed by Yáñez-Macias et al. [22]. This is an aqueous suspension of spherical nanoparticles $(\varnothing<, 90 \mathrm{~nm})$ formed by the self-assembly of a diblock copolymer, comprising a cationic hydrophilic block and a hydrophobic block. It is hypothesized that optimal combinations of the nanoparticle size, aggregation number, and cationic and lipophilic natures of the proposed PNBs might improve their diffusion within the plant and abridge the way to reach pathogen-housing sites. Our preliminary results using these PNBs to fight cosmopolitan bacteria in vitro were promising [23]. Thus, our understanding of these nanomaterials and their dynamics in plants' vascular systems led to testing smaller polymeric nanospheres based on cationic poly $(N, N$-dimethylaminoethyl methacrylate) diblock copolymers. The results of the initial trials using such PNBs to combat $\mathrm{CaLso}$ are reported here. It is worth mentioning that the proposed PNB can be applied in a foliar fashion, especially airborne and in small dosages, and on every kind of vegetable crop.

\section{Results}

\subsection{Nanobactericide Preparation}

The original synthetic route [22] grounded on the well-known capacity of amphiphilic block copolymers to self-assemble [24,25] was slightly modified to prepare PNB in three steps, starting from a PDMAEMA macroRAFT agent: this is (a) the synthesis of poly $(\mathrm{N}, \mathrm{N}$ - 
dimethylaminoethyl methacrylate-statistical-butyl methacrylate) (P(DMAEMA-stat-BMA)) copolymer by reversible addition-fragmentation chain transfer (RAFT) polymerization, (b) quaternization of the tertiary amine pendant groups in the backbone of the copolymer, and (c) self-assembly of the block copolymer in aqueous media to induce the formation of cationic nanoparticles through PISA. The synthesized statistical copolymer was characterized by $1 \mathrm{H}$ NMR spectroscopy (see Figure S1). The characteristic signals ascribed to the DMAEMA and BMA monomeric units are visible in the spectrum. Subsequently, the pendant amino groups of the copolymer were treated with an excess of methyl iodide to form quaternary ammonium groups, which are ultimately responsible for interacting with bacteria. The degree of quaternization was corroborated by the disappearance of the proton signal of DMAEMA in the $1 \mathrm{H}$ NMR spectrum $(\delta=2.58$ and $2.30 \mathrm{ppm})$ as observed in Figure S2 in Supplementary Materials.

The PISA approach was used to prepare polymeric nanoparticles (or PNB). DLS investigations suggested that well-defined nanoparticles of ca. $50 \mathrm{~nm}$ with a narrow size distribution ( 0.09) and a low polydispersity index (PDI) value were obtained (Figure 1). Figure $1 \mathrm{a}, \mathrm{b}$ shows the spherical shape and the size uniformity of the obtained nanoparticles. The results from the DLS analysis can be associated with the data obtained from the $\zeta$ measurements, which is a parameter that determines the attraction or repulsion forces between particles. The higher the absolute value of $\zeta$, the stronger the repulsion between particles, which increases the colloidal system's stability. At low $\zeta$ values, van der Waals attractive forces dominate the repulsion forces, resulting in particle aggregation. In general, stable colloidal suspensions show $\zeta$ values greater than $\pm 25 \mathrm{mV}$ [26]. A $\zeta$ value of $50.2 \pm 6.1$ was obtained in our study. It is important to point out that regardless of the absolute value obtained in $\zeta$ measurements, all the investigated latexes showed a positive value, which indicates the presence of the quaternary groups on the surface of nanoparticles.
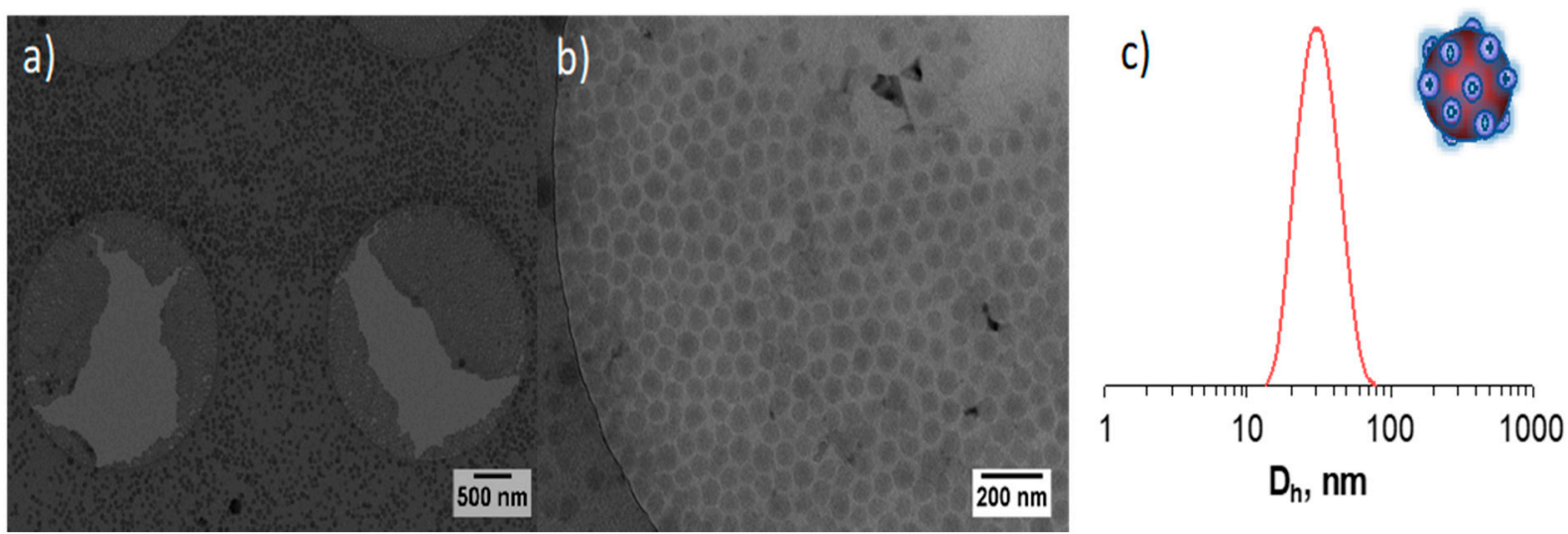

Figure 1. Cryo-TEM image (a,b) of polymeric nanoparticles in solution showing the formation of spheres. (c) Hydrodynamic size distribution of polymeric nanoparticles.

To corroborate the nanoparticle size estimated by DLS analysis and to further investigate their morphology, polymeric nanoparticles were examined by cryo-TEM. This technique allows observing the morphology of materials in solution with nanometer spatial resolution and a temporal resolution of $<1 \mathrm{~s}$. The micrography (Figure 1) revealed the presence of spherical nanoparticles in the range of $40-60 \mathrm{~nm}$ with a uniform size distribution, which is in good agreement with the size determined by DLS measurements.

\subsection{Effect of Different Doses of the PNB in Tomato Plants Infected by CaLso}

Beforehand, samples of the infested plants and psyllids were subjected to PCR analysis to confirm the presence of $\mathrm{CaLso}$. The obtained sequences shared the identity of Candidatus Liberibacter solanacearum outer membrane protein gene to the extent of $98-100 \%$ [8]. 
In the first bioassay, infected plants treated with dispersions of different concentrations of PNB showed differences in symptoms at the evaluation time of 25 days (Figure 2). Tomato plants treated with PNB concentrations of 40 and $70 \mathrm{ppm}$ presented reduced signs of chlorosis and necrosis as compared to untreated plants, though plants treated with a solution containing $20 \mathrm{ppm}$ of PNB showed moderate chlorosis. Since most of the plants treated with a PNB concentration of $70 \mathrm{ppm}$ showed a reduction in typical symptoms produced by $\mathrm{CaLso}$, this concentration was selected for the subsequent bioassays. As expected, control plants did not present any symptoms of $\mathrm{CaLso}$, but when these healthy plants were exposed to the PNB dispersion (of $70 \mathrm{ppm}$ concentration), a smaller plant size was detected compared to those taken as absolute controls (see Figure S3). To evaluate differences in CaLso abundance among the different treatments, DNA from all samples was analyzed by qPCR. The relative quantification of $\mathrm{CaLso}$ was determined for untreated plants and those treated with different PNB doses. The latter showed higher statistical differences as compared to the untreated cases with $p<0.0001$ (Figure 3). qPCR results also showed the presence of higher pathogen levels in untreated samples (Figure 3).

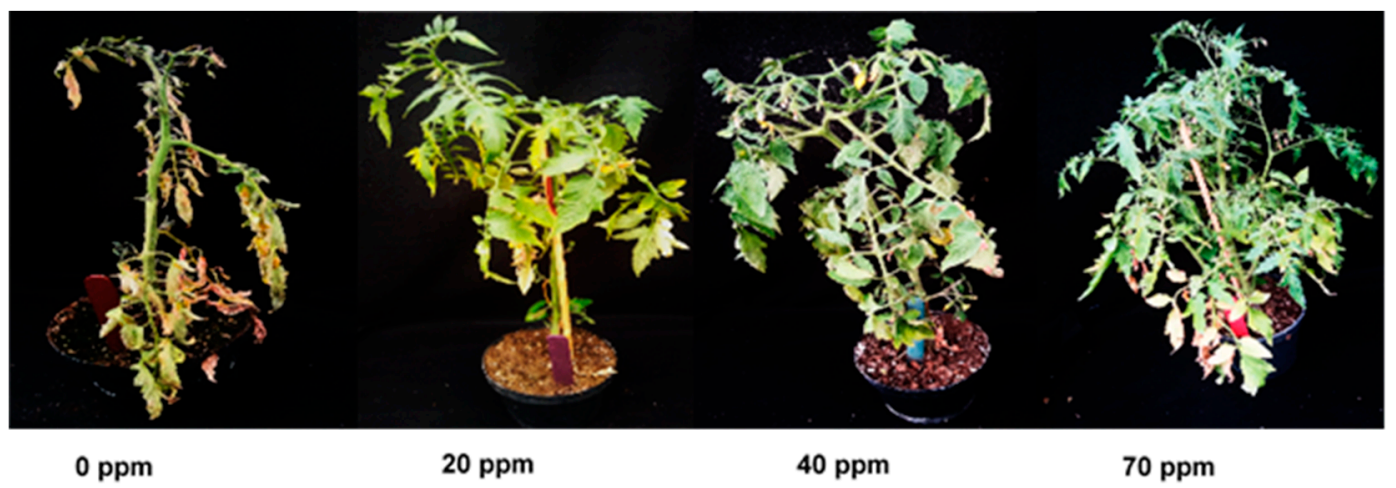

Figure 2. Tomato plants (Solanum lycopersicum cv. Floradade) infected with the Candidatus Liberibacter solanacearum (CaLso) treated with different doses of PNB. Evaluated at 25 days post-treatment.

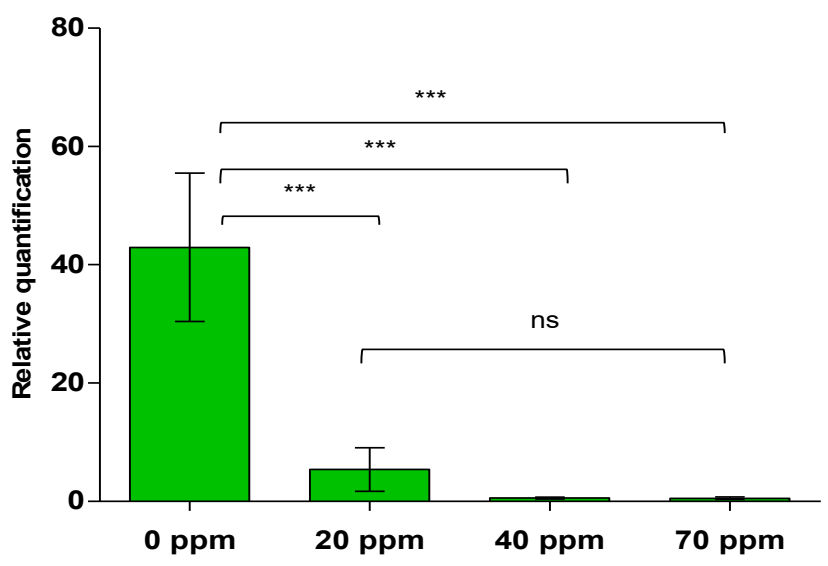

Figure 3. Quantification of the Candidatus Liberibacter solanacearum (CaLso) in tomato leaves (Solanum lycopersicum) using qPCR. Differences were detected in three dose levels of PNB (i.e., dispersions containing 20,40, and $70 \mathrm{ppm}$ of PNB). Values are means \pm SE $(n=9)$. Level of significance: ${ }^{* * *}, p<0.001$; ns, no significance. One-way ANOVA, Tukey's multiple comparison test at $p \leq 0.05$.

\subsection{Time of Efficiency in CaLso Reduction by PNB Treatment}

When the three doses of PNB were evaluated in infected plants, a reduction in symptoms for plants treated with a dispersion of 70 ppm PNB was clearly evident. Therefore, to assess the efficiency of the PNB at a concentration of $70 \mathrm{ppm}$, symptoms in the tomato plants were monitored at 7 days post-treatment (Figure S4). No symptomatic differences 
were observed. CaLso abundance was also assessed, and no significant differences were detected in these treated plants (Figure 4a). CaLso abundance showed low levels in assays involving PNB treatments, but it was not sufficient to consider a significant difference $(p=0.1778)$.

a)

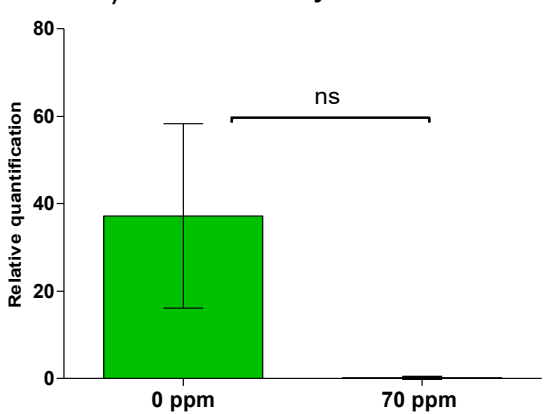

b)

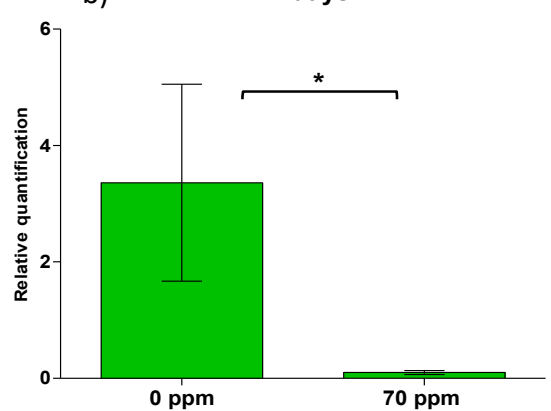

c)

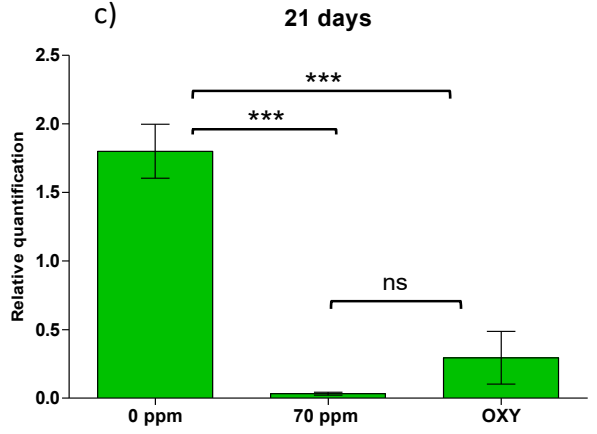

Figure 4. Quantification of the Candidatus Liberibacter solanacearum (CaLso) in tomato leaves (Solanum lycopersicum) at (a) 7 days, (b) 14 days, and (c) 21 days post-treatment with 70 ppm of PNB using qPCR. Response to treatment with oxytetracycline (OXY) is shown in (c). Values are means $\pm \mathrm{SE}(n=6)$; Level of significance: ${ }^{*}, p<0.05 ;{ }^{* * *}, p<0.001 ; \mathrm{ns}$, not significant. One-way ANOVA test at $p \leq 0.05$.

For those plants evaluated at 14 days post-treatment, symptomatic differences were detected, and chlorosis was evident in untreated tomato plants as compared to plants treated with the PNB dispersion containing $70 \mathrm{ppm}$ (Figure S5). In this case, CaLso reductions at 14 days showed significant differences $(p=0.0253)$ (Figure $4 b)$.

For plants evaluated at 21 days post-treatment, symptomatic differences were detected among plants subjected to the different treatments; chlorosis was evident in $\mathrm{CaLso}$ tomato plants, as well as in those treated with oxytetracycline, whereas tomato plants treated with PNB showed reduced CaLso symptoms (Figure S6). Thus, the effectivity of PNB dispersion was evident at 21 days post-treatment and resulted in a significant statistical difference (Figure 4c). Furthermore, the antibiotic oxytetracycline also presented significant differences, although the CaLso symptoms were more visible than in those treatments using PNB.

\section{Discussion}

All control strategies for phloem bacteria such as CaLso are mainly focused on psyllid extermination. These strategies include different insecticide formulations $[6,27,28]$, releasing parasitoids (Tamarixia triozae) [12], and using plant extracts [28]. The psyllid colonies decline when any of these approaches are used, which could represent one advantage but is not a determinant for $\mathrm{CaLso}$ reduction inside the host plant. Moreover, current alternatives for CaLso removal in host plants are quite limited.

This research is not directed to insect control but the pathogenic microorganism itself. It is worth noting that this study was carried out at a greenhouse level but can be seen as a viable alternative for reducing the use of agrochemicals in open fields. Accessorily, it results in better yields of solanaceous plants. In this sense, it was decided to investigate the effect of our water dispersion of PNB ( $\sim 50 \mathrm{~nm}$ in size) in plants infected with CaLso. Initially, uninfected plants were treated with PNB to search for adverse effects. Remarkably, these plants do not show either visual adverse effects or growth promotion compared to untreated plants (Figure S3). Although experiments to evaluate oxidative burst (reactive oxygen species levels) were not carried out in plants treated with PNB, they were grown generally for three weeks.

Moreover, when different PNB doses were sprayed in infected plants, a significant reduction in symptoms was observed, especially when a PNB dispersion of $70 \mathrm{ppm}$ was used (Figure 2). Later, these plants presented a reduced CaLso abundance that was statistically 
contrasting between the different investigated PNB doses (i.e., 20 ppm (87.45\%), 40 ppm $(98.68 \%)$, and $70 \mathrm{ppm}(98.8 \%)$ ) (see Figure 3). Although reduced CaLso abundances were observed in PNB dispersions of 40 and 70 ppm, the selected concentration was $70 \mathrm{ppm}$ for a subsequent bioassay to determine the extent of such effect. For that, the bacterial load level was assessed after 25 days post-treatment (Figure 2).

For a second bioassay, plants infected were evaluated for three weeks $(7,14$, and 21 days post-treatment). This experiment aimed to determine the time of the effectivity of the treatment with $70 \mathrm{ppm}$ PNB dispersion. In plants evaluated at 7 days, no statistically significant differences were detected (Figure 4a); plants showed similar symptoms of chlorosis in infected plants and those treated with PNB (70 ppm) (Figure S4). CaLso abundance was reduced for treatments with PNB dispersions of $70 \mathrm{ppm}$ treatment, although no significate differences were detected between treatments (Figure 4a). Surprisingly, significant differences were observed in infested plants treated with the PNB dispersion of 70 ppm observed after 14 days. Indeed, pathogen abundance was reduced (ca. 98.96\% (see Figure 4b)). A significant reduction in CaLso two weeks after the PNB application was detected (see Figure S5). Finally, plants evaluated after three weeks showed statistical differences between the treatments (Figures $4 \mathrm{c}$ and S6).

On the flip side, oxytetracycline was less effective at $100 \mathrm{ppm}$ for 30 days with bacterial titer reduction of ca. 50\% [17,27,29]. The treatment with the PNB dispersion of $70 \mathrm{ppm}$ achieved pathogen reduction of ca. $97.78 \%$, while oxytetracycline showed ca. $79.83 \%$ pathogen reduction.

Moreover, plants treated with a PNB dispersion of $70 \mathrm{ppm}$ in this bioassay showed a reduced flower abortion of ca. $60 \%$ (data not shown). This could be correlated to the reduced $\mathrm{CaLso}$ abundance detected via qPCR evaluations. Using this kind of PNB for solanaceous crops in open fields or greenhouse conditions might increase crop yields and even be conceivably effective for controlling the HLB disease in citrus trees. The mechanism of action of the PNB material in these plants remains unknown. However, we speculated that the cationic properties of investigated PNB material could promote interactions with the $\mathrm{CaLso}$ cell membrane and lyse the bacteria to cause its death. In this regard, to the best of our knowledge, the use of this kind of nanomaterial to combat $\mathrm{CaLso}$ in plants is very scarcely described in the literature.

The biodegradation of PNB is a subject of enormous concern. For that reason, the design of PNB includes well-known monomers in terms of toxicity. As a reference, homopolymerizing BMA poly(BMA) is obtained, a material widely used in many fields. Poly(BMA) is highly biocompatible, $100 \%$ recyclable, and non-biodegradable. Thus, a large amount of hydroxyl propyl methacrylate (HPMA) was included to constrain the non-biodegradability. Poly(HPMA) is widely used in cosmetology since it shows good hydrophilicity, biocompatibility, etc. Both monomers together in the core of PNB give a chance to make the material apt to decompose in nature in the long term. Concerning the active part of PNB, i.e., the corona of quaternary amine ions, they are covalently anchored to the particle. Their structure is similar to some commercial bactericides currently used in agriculture, such as oxytetracycline.

It is worth mentioning that during the experimentation, no alarm symptoms were detected concerning the health of the uninfected plants treated within the first 25 days after the first contact with PNB. Therefore, no analysis or treatment was required. It is also important to note that the PNB treatment reduced the bacterial population in tomato plants. Upon total DNA extraction in infected tomato leaves, a low presence of DNA from the pathogen was detected when the qPCR analysis was performed.

\section{Materials and Methods}

\subsection{Material}

4,4'-Azobis(4-cyanovaleric acid) (ACVA), 4-cyano-4-(phenyl-carbonothioylthio) pentanoic acid (CPADB), and methyl iodide (MeI) were obtained from Aldrich and used as received. The monomers $N, N$-dimethylaminoethyl methacrylate (DMAEMA), butyl 
methacrylate (BMA), and hydroxypropyl methacrylate (HPMA) were purified by stirring in the presence of inhibitor remover for hydroquinone (Aldrich) for 30 min before usage.

\subsection{Synthesis of Nanobactericide}

The original synthetic route [22] was modified to prepare PNB in three steps: (a) the synthesis of a poly $(N, N$-dimethylaminoethyl methacrylate-statistical-butyl methacrylate) (P(DMAEMA-stat-BMA)) copolymer by reversible addition-fragmentation chain transfer (RAFT) polymerization, (b) quaternization of the tertiary amine pendant groups in the backbone of the copolymer, and (c) self-assembly of the block copolymer in aqueous media to induce the formation of cationic nanoparticles.

\subsection{RAFT Synthesis of P(DMAEMA-stat-BMA) Copolymer}

A round-bottom flask was charged with DMAEMA (4.52 g, $28.8 \mathrm{mmol})$, BMA (1.02 g, $7.2 \mathrm{mmol})$, CPADB (0.134 g, $0.48 \mathrm{mmol}), \operatorname{ACVA}(0.041 \mathrm{~g}, 0.144 \mathrm{mmol})$, and ethanol $(9.00 \mathrm{~g})$. The sealed reaction vessel was purged with nitrogen and placed in a preheated oil bath at $70{ }^{\circ} \mathrm{C}$ for $6 \mathrm{~h}$. The obtained P(DMAEMA-stat-BMA) was purified by diluting the reaction mixture with tetrahydrofuran (THF) and precipitated in cold hexane.

\subsection{Quaternization of $P(D M A E M A$-stat-BMA) Copolymer}

The quaternization of P(DMAEMA-stat-BMA) copolymer was carried out using methyl iodide following a procedure reported elsewhere [30], which was modified to achieve a higher degree of quaternization and yielded a poly((2-[methacryloyloxy]ethyl) trimethylammonium iodide-stat-BMA) (P(METAI-stat-BMA)) copolymer denoted as QCOP.

\subsection{Synthesis of Nanobactericide as a Nanoparticle by Polymerization-Induced Self-Assembly (PISA)}

HPMA (1.40 g, $9.74 \mathrm{mmol}), \operatorname{ACVA}(0.018 \mathrm{~g}, 0.006 \mathrm{mmol})$, and Q-COP (0.150 g, $0.020 \mathrm{mmol})$ were dissolved in a buffer solution $\mathrm{pH}=6.0(6.27 \mathrm{~g})$ to prepare an aqueous dispersion polymerization at $20 \mathrm{wt}$ \% solids. This reaction mixture was sealed in a round-bottom flask containing a magnetic stirring bar, purged with nitrogen for $15 \mathrm{~min}$, and then placed in a preheated oil bath at $70{ }^{\circ} \mathrm{C}$ for $3 \mathrm{~h}$ under moderate stirring (Scheme 1).
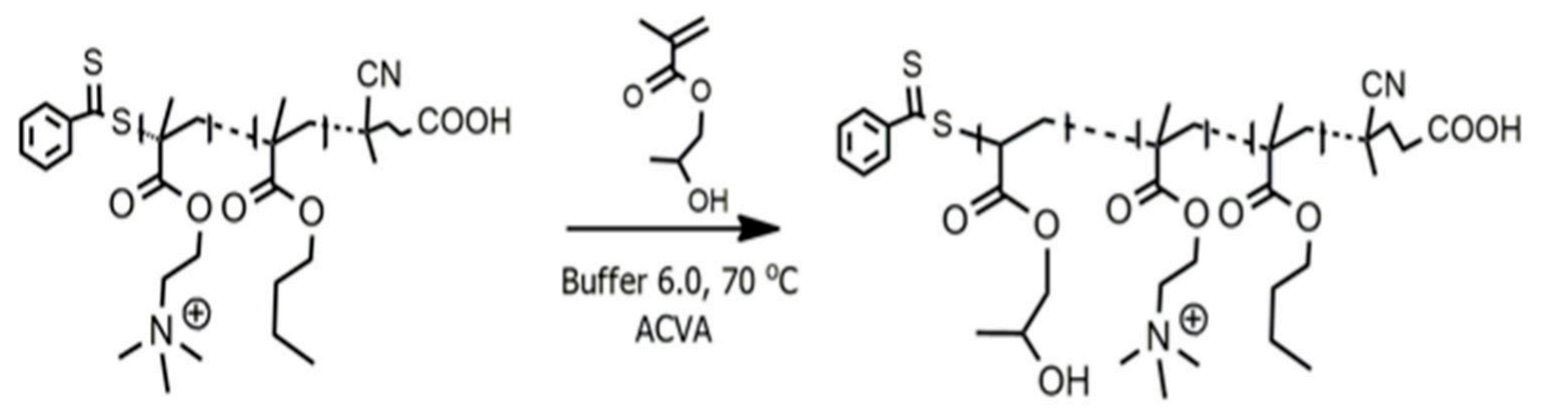

Scheme 1. Schematic representation of synthetic route to prepare PNB.

\subsection{Polymer Characterization Methods}

Proton nuclear magnetic resonance $\left({ }^{1} \mathrm{H}\right.$ NMR) spectra were recorded at room temperature on a $300 \mathrm{MHz}$ Bruker Avance NMR spectrometer using deuterated chloroform $\left(\mathrm{CDCl}_{3}\right)$ or deuterated dimethyl sulfoxide (DMSO- $\left.\mathrm{d}_{6}\right)$ as solvent.

Dynamic light scattering (DLS) measurements were performed using a Malvern Instrument Zetasizer Nano Series instrument equipped with a $4 \mathrm{~mW}$ He-Ne laser operating at $633 \mathrm{~nm}$. The scattered light was detected at an angle of $173^{\circ}$. Each measurement was performed in triplicate. The mean particle size was calculated by applying the nonlinear least-squares fitting mode. 
Electrophoretic light scattering was used to measure zeta potential $(\zeta)$. The measurements were carried out in a Zetasizer Nano ZS by applying Doppler velocimetry. The analysis was performed in triplicate at $25{ }^{\circ} \mathrm{C}$. According to the Henry equation, $\zeta$ was calculated from the electrophoretic mobility $(\mu)$.

For cryogenic transmission electron microscopy (cryo-TEM) investigations, samples were rapidly blotted and plunged into a cryogenic reservoir containing liquid ethane. After preparation, prepared samples were stored and measured at a temperature below $-176{ }^{\circ} \mathrm{C}$ to avoid crystalline ice layers.

\subsection{Plant Growth Conditions and Insects}

Tomato seeds (Solanum lycopersicum cv. Floradade, Crown Seeds, Homestead, FL, USA) were germinated in peat moss until 3-4 leaves were presented. Then, tomato seedlings were transplanted into 1.5 L pots containing peat moss and perlite $(70: 30 \mathrm{v} / \mathrm{v})$. The plants were watered every three days and fertilized once a week with $20 \mathrm{~N}-20 \mathrm{P}-20 \mathrm{~K}$ and microelements solution (FertiDrip, Agrodelta, Monterrey, Mexico). All plants were maintained under greenhouse conditions at $28 \pm 2{ }^{\circ} \mathrm{C}$ with relative humidity $(\mathrm{RH})=45 \%$ and regularly monitored until they reached 6 to 7 fully extended leaves before bioassays.

The tomato psyllids (Bactericera cockerelli) CaLso-positive were reared on tomato plants under greenhouse conditions [31] to induce infestation. Control plants were kept separated to avoid infestation.

\subsection{Plant Treatments}

Bioassays were performed using 4-day-old Bactericera cockerelli adults. Individual tomato plants, covered with an antiaphid mesh, were infested with 30 unsexed adults, and the insect-plant interaction was allowed for two days. After that, B. cockerelli adults were removed from the plants by gentle aspiration. Next, plants were tested through polymerase chain reaction (PCR) to verify infection. The bioassays were carried out only for $\mathrm{CaLso-positive} \mathrm{plants} \mathrm{and} \mathrm{their} \mathrm{respective} \mathrm{controls.}$

A first bioassay was performed in November-December 2018 in Saltillo, Mexico, to evaluate the effect of using different doses of PNB in infected tomato plants (nine plants per dosage were utilized). These investigations included infected plants treated with 70 , 40, or 20 ppm of PNB and its corresponding controls. Additionally, uninfected plants were treated with 70 ppm of PNB as a second control.

Each plant was initially sprayed with $3 \mathrm{~mL}$ of the corresponding PNB concentration, followed by a respray one week later. After that, all plants were kept in separate tunnels with antiaphid mesh to avoid $B$. cockerelli reinfestation. The plants were grown under greenhouse conditions and were monitored every week for symptoms. Finally, 25 days post-treatment, leaves were collected, flash-frozen in liquid nitrogen, ground in a mortar and pestle, and stored at $-80{ }^{\circ} \mathrm{C}$ until needed for deoxyribonucleic acid (DNA) isolation.

A second bioassay was performed in March-April 2019. Infected tomato plants were separately treated with two different bactericides. For PNB, plants were initially sprayed and resprayed at day 7 with a solution containing $70 \mathrm{ppm}$ of PNB. After this treatment, plants were allowed to grow and appraised every week for three weeks to estimate an adequate treatment interval $(7,14$, or 21 days). This experiment was performed with a series of 18 plants, including 18 (untreated) control plants. For comparison, 18 plants were treated with a solution containing $600 \mathrm{ppm}$ of oxytetracycline (second bactericide) and evaluated 21 days after treatment. For both bioassays, plants were allowed to grow under similar conditions after their respective treatment and were monitored for symptoms. Finally, leaves were collected, flash-frozen in liquid nitrogen, and stored at $-80{ }^{\circ} \mathrm{C}$ until needed for DNA isolation.

\subsubsection{DNA Isolation}

Composite DNA samples were obtained from the combined and isolated DNA from leaves from each treatment in both bioassays. DNA isolation was performed using the 
DNeasy Plant Mini Kit (QIAGEN, Hilden, Germany), according to the manufacturer's instructions, treated with DNAase-free RNAase.

The samples were separated on $1.2 \%$ agarose gels (Certified Molecular Biology Agarose, Bio-Rad Laboratories, Hercules, CA, USA) to verify DNA isolation quality. All DNA samples were quantified using the ND-1000 Spectrophotometer (NanoDrop Products, Wilmington, DE, USA).

\subsubsection{Endpoint and Quantitative Real-Time PCR}

Endpoint PCR was used to verify the presence of $C a$ Lso in DNA isolated from infested tomato plants and B. cockerelli adults. Candidatus Liberibacter Zebra Chip (CLZC) specific primers were used by following a protocol described elsewhere [8]: CLZC-F $5^{\prime}$ -

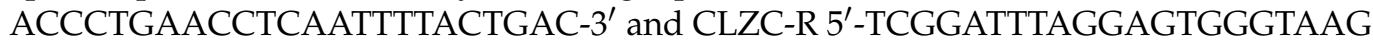
TGG-3'. All PCR amplifications were performed using Taq PCR Master Mix Kit (QIAGEN, Hilden, Germany) under the following conditions: 1 cycle at $94{ }^{\circ} \mathrm{C}$ for $3 \mathrm{~min} ; 35$ cycles at $94{ }^{\circ} \mathrm{C}$ for $45 \mathrm{~s}, 55^{\circ} \mathrm{C}$ for $45 \mathrm{~s}$, and $72{ }^{\circ} \mathrm{C}$ for $45 \mathrm{~s}$; followed by a final process at $72{ }^{\circ} \mathrm{C}$ for $5 \mathrm{~min}$. Two amplicons were obtained from infested plants and insects ( 185 bp), separated on $2 \%$ agarose gels, and purified using QIAquick Gel Extraction Kit (QIAGEN, Hilden, Germany). Next, these fragments were quantified and sequenced (ELIM Biopharmaceuticals, Inc., Hayward, CA, USA). The obtained sequences were compared with the aid of the database from the National Center for Biotechnology Information, USA. Genomic DNA was isolated from leaves of 5-week-old healthy plants (control), PNB-treated plants, and infected tomato plants (CaLso and CaLso PNB-treated plants). Quantitative real-time PCR (qPCR) was employed to detect $\mathrm{CaLso}$ differences in all treatments during the bioassays. CLZC specific primers were used for CaLso detection, and RPL2 (Tomato Ribosomal Protein L2) was used as an endogenous reference gene [9], employing the following primers: RPL2-F $5^{\prime}$ GAGGGCGTACTGAGAAACCA-3' and RPL2-R 5'-CTTTTGTCCAGGAGGTGCAT- $3^{\prime}$. The analysis was performed using iQ SYBR Green Supermix (Bio-Rad Laboratories, Inc., Hercules, CA, USA), CLZC and RPL2 primers at $300 \mathrm{nM}$, and $100 \mathrm{ng}$ of genomic DNA using Hard-Shell PCR Plates (Bio-Rad Laboratories, Inc., Hercules, CA, USA), according to the manufacturer's protocol. All qPCR analyses were achieved on a CFX96 Touch RealTime PCR Detection System (Bio-Rad Laboratories, Inc., Hercules, CA, USA). For the first bioassay, six treatments with nine biological samples with three technical replicates per sample were used for qPCR investigations. The second bioassay was carried out with five treatments and three control treatments with six biological samples with three technical replicates per sample. The qPCR was performed under the following conditions: 1 cycle at $95^{\circ} \mathrm{C}$ for $10 \mathrm{~min}, 35$ two-step cycles each at $95^{\circ} \mathrm{C}$ for $15 \mathrm{~s}$ and $55^{\circ} \mathrm{C}$ for $60 \mathrm{~s}$, and melting curve of $65-95{ }^{\circ} \mathrm{C}$ for $5 \mathrm{~s}$. Data were obtained with CFX Manager Version 3.1 Software (Bio-Rad Laboratories, Inc., Hercules, CA, USA). Quantification of CaLso in the infected plants vs. control plants was obtained relative to the endogenous RPL2 gene according to the comparative $C_{t}$ method $\left(2^{-\Delta \Delta C t}\right)[32]$.

\subsection{Data Analysis}

All biological samples were analyzed in triplicate, and average values $\left(2^{-\Delta \Delta C t}\right)$ were calculated for each specimen. One-way ANOVA was used to analyze data from all bioassays, and Tukey's multiple comparison test was performed to obtain all possible pairwise differences of means. Statistical analyses were performed using GraphPad Prism version 5 for Windows (GraphPad Software, La Jolla, CA, USA).

\section{Conclusions}

Candidatus Liberibacter solanacearum ( $\mathrm{CaLso})$ is considered one of the most economically important pathogens in solanaceous crops, principally in potatoes and tomatoes. Because $\mathrm{CaLso}$ is phloem-limited, it represents a challenge for the design of practical control strategies. In this contribution, we reported the antibacterial activity of a polymeric nanobactericide (PNB) in tomato plants against $\mathrm{CaLso}$. We demonstrated that evenly sized 
bactericide particles (50-70 ppm) could reduce the presence of the pathogen in tomato leaves by up to $98.96 \%$. We also showed that control plants treated with a PNB dispersion did not display phytotoxicological effects. Finally, we believe that further optimizations can be made around this concept to obtain even more effective nanobactericide materials for fighting phloem-obligated pathogens. For instance, PNB could be combined with other bioactive materials to imprint a synergistic effect to expand its antimicrobial activity to other crops.

Supplementary Materials: The following are available online at https:/ /www.mdpi.com/article/ 10.3390/plants10102096/s1, Figure S1: 1H NMR spectrum of P(DMAEMA-co-BMA), recorded in $\mathrm{CDCl}$, with peak assignments. Inset is an expanded region highlighting the presence of the -RAFT end group protons. Figure S2: 1H NMR spectrum of P(METAI-co-BMA), recorded in DMSO-d6. Figure S3: Healthy plants of tomato (Solanum lycopersicum) at 21 days of treatment with $70 \mathrm{ppm}$ of PNB (PNB) and water treated (Control). Plants were grown in greenhouse conditions in absence of Bactericera cockerelli and Candidatus Liberibacter solanacearum. Figure S4: Infected plants of tomato (Solanum lycopersicum) with Candidatus Liberibacter solanacearum (CaLso) at 7 days of treatment with 70 ppm of PNB. Figure S5: Infected plants of tomato (Solanum lycopersicum) with Candidatus Liberibacter solanacearum ( $\mathrm{CaLso}$ ) at 14 days of treatment with 70 ppm of PNB. Figure S6: Infected plants of tomato (Solanum lycopersicum) with Candidatus Liberibacter solanacearum (CaLso) at 21 days of treatment with $70 \mathrm{ppm}$ of PNB and oxytetracycline.

Author Contributions: A.N.G.-S. performed all biological experiments and qPCR analysis; R.Y.-M. and C.G.-S. synthesized nanobactericide; R.G.-S. and J.H.V.-S. designed experiments and wrote the manuscript; J.L.H.-F. and A.Á.-M. analyzed the qPCR data. All authors have read and agreed to the published version of the manuscript.

Funding: The authors kindly acknowledge CONACYT (México) for funding this work through the FORDECYT project 296356. C.G.S. thanks the Center for Excellence "PolyTarget" (SFB 1278 (316213987), projects B02 and Z01) of the Deutsche Forschungsgemeinschaft (DFG, Germany) for financial support.

Institutional Review Board Statement: Not applicable.

Informed Consent Statement: Not applicable.

Data Availability Statement: Data is contained within the article or supplementary material.

Acknowledgments: Authors acknowledge the financial support provided by Centro de Investigación en Química Aplicada (CIQA), Grant No. 6495/6510/6627. This work was also partially funded by the Consejo Nacional de Ciencia y Tecnología (CONACyT) (grant number 636640) (Cátedras-CONACyT No. 1333 to JHVS). Authors thank Alberto Flores Olivas for the donation of a sample of Bactericera cockerelli and fulfilling discussions of results. RYM thanks the support of CONACYT through the research grant 315878. The valuable technical support of Judith Cabello (CIQA) during the synthesis of the PNB material is acknowledged.

Conflicts of Interest: The authors declare no conflict of interest.

\section{References}

1. Ghatak, A.; Chaturvedi, P.; Paul, P.; Agrawal, G.K.; Rakwal, R.; Kim, S.T.; Weckwerth, W.; Gupta, R. Proteomics survey of Solanaceae family: Current status and challenges ahead. J. Proteom. 2017, 169, 41-57. [CrossRef] [PubMed]

2. Salim, M.M.R.; Rashid, M.H.; Hossain, M.M.; Zakaria, M. Morphological characterization of tomato (Solanum lycopersicum L.) genotypes. J. Saudi Soc. Agric. Sci. 2018, 19, 233-240. [CrossRef]

3. WANG, Y.; ZHANG, Y.; GAO, Z.; YANG, W. Breeding for Resistance to Tomato Bacterial Diseases in China: Challenges and Prospects. Hortic. Plant J. 2018, 4, 193-207. [CrossRef]

4. Fennell, J.T.; Fountain, M.T.; Paul, N.D. Direct effects of protective cladding material on insect pests in crops. Crop Prot. 2019, 121, 147-156. [CrossRef]

5. Blancard, D. The Tomato Plant and its Culture. In Tomato Diseases, 2nd. ed; Academic Press: San Diego, CA, USA, $2012 ;$ pp. 17-34. ISBN 978-0-12-387737-6.

6. Munyaneza, J.E. Zebra Chip Disease of Potato: Biology, Epidemiology, and Management. Am. J. Potato Res. 2012, 89, 329-350. [CrossRef] 
7. Sengoda, V.G.; Cooper, W.R.; Swisher, K.D.; Henne, D.C.; Munyaneza, J.E. Latent Period and Transmission of “Candidatus Liberibacter solanacearum" by the Potato Psyllid Bactericera cockerelli (Hemiptera: Triozidae). PLoS ONE 2014, 9 , e93475. [CrossRef]

8. Crosslin, J.M.; Lin, H.; Munyaneza, J.E. Detection of 'Candidatus Liberibacter Solanacearum' in the Potato Psyllid, Bactericera cockerelli (Sulc), by Conventional and Real-Time PCR. Southwest. Entomol. 2011, 36, 125-135. [CrossRef]

9. Avila, C.A.; Arévalo-Soliz, L.M.; Jia, L.; Navarre, D.A.; Chen, Z.; Howe, G.A.; Meng, Q.-W.; Smith, J.E.; Goggin, F.L. Loss of function of FATTY ACID DESATURASE7 in tomato enhances basal aphid resistance in a salicylate-dependent manner. Plant Physiol. 2012, 158, 2028-2041. [CrossRef]

10. Liu, J.-F.; Zhang, Z.-Q.; Beggs, J.R.; Paderes, E.; Zou, X.; Wei, X.-Y. Lethal and sublethal effects of entomopathogenic fungi on tomato/potato psyllid, Bactericera cockerelli (Šulc) (Hemiptera: Triozidae) in capsicum. Crop Prot. 2020, 129, 105023. [CrossRef]

11. Lacey, L.A.; Liu, T.-X.; Buchman, J.L.; Munyaneza, J.E.; Goolsby, J.A.; Horton, D.R. Entomopathogenic fungi (Hypocreales) for control of potato psyllid, Bactericera cockerelli (Šulc) (Hemiptera: Triozidae) in an area endemic for zebra chip disease of potato. Biol. Control 2011, 56, 271-278. [CrossRef]

12. De Lourdes Ramírez-Ahuja, M.; Rodríguez-Leyva, E.; Lomeli-Flores, J.R.; Torres-Ruiz, A.; Guzmán-Franco, A.W. Evaluating combined use of a parasitoid and a zoophytophagous bug for biological control of the potato psyllid, Bactericera cockerelli. Biol. Control 2017, 106, 9-15. [CrossRef]

13. Pérez-Aguilar, D.A.; Martínez, A.M.; Viñuela, E.; Figueroa, J.I.; Gómez, B.; Morales, S.I.; Tapia, A.; Pineda, S. Impact of the zoophytophagous predator Engytatus varians (Hemiptera: Miridae) on Bactericera cockerelli (Hemiptera: Triozidae) control. Biol. Control 2019, 132, 29-35. [CrossRef]

14. Tamayo-Mejía, F.; Tamez-Guerra, P.; Guzmán-Franco, A.W.; Gomez-Flores, R. Can Beauveria bassiana Bals. (Vuill) (Ascomycetes: Hypocreales) and Tamarixia triozae (Burks) (Hymenoptera: Eulophidae) be used together for improved biological control of Bactericera cockerelli (Hemiptera: Triozidae)? Biol. Control 2015, 90, 42-48. [CrossRef]

15. McVay, J.; Sun, X.; Jones, D.; Urbina, H.; Aldeek, F.; Cook, J.M.; Jeyaprakash, A.; Hodges, G.; Smith, T. Limited persistence of residues and metabolites in fruit and juice following penicillin trunk infusion in citrus affected by Huanglongbing. Crop Prot. 2019, 125, 104753. [CrossRef]

16. Kranjc, E.; Drobne, D. Nanomaterials in Plants: A Review of Hazard and Applications in the Agri-Food Sector. Nanomaterials 2019, 9, 1094. [CrossRef]

17. Nwugo, C.C.; Lin, H.; Duan, Y.; Civerolo, E.L. The effect of 'Candidatus Liberibacter asiaticus' infection on the proteomic profiles and nutritional status of pre-symptomatic and symptomatic grapefruit (Citrus paradisi) plants. BMC Plant Biol. 2013, 13, 59. [CrossRef] [PubMed]

18. Puttamuk, T.; Zhang, S.; Duan, Y.; Jantasorn, A.; Thaveechai, N. Effect of chemical treatments on 'Candidatus Liberibacter asiaticus' infected pomelo (Citrus maxima). Crop Prot. 2014, 65, 114-121. [CrossRef]

19. Nelson, R. Controversial antibiotic use for crops approved in Florida. Lancet Infect. Dis. 2019, 19, 697-698. [CrossRef]

20. Kumar, D.; Braun, C.; Terrazas Portillo, J.C.; Tapia Ramos, E.; Cambero Ramírez, L.M. Use of Isotianil for Control of Zebra Chip Disease. U.S. Patent Application No. WO/2017/055342, 2017.

21. Yañez-Macías, R.; Muñoz-Bonilla, A.; De Jesús-Tellez, A.M.; Maldonado-Textle, H.; Guerrero-Sánchez, C.; Schubert, S.U.; Guerrero-Santos, R. Combinations of Antimicrobial Polymers with Nanomaterials and Bioactives to Improve Biocidal Therapies. Polymers 2019, 11, 1789. [CrossRef]

22. Yañez-Macías, R. Study of the Structure-Property Relationship of Antibacterial and Thermo-Responsive Copolymers Synthesized by RAFT Polymerization. Ph.D. Thesis, Centro de Investigación en Química Aplicada, Saltillo, Coahuila, Mexico, 2017.

23. Guerrero-Santos, R.; Valenzuela-Soto, J.H.; Yañez-Macías, R.; García-Sánchez, A.N.; Lugo de León, P. Process for Obtaining and Using a Phytoformulation with Dual Action to Eliminate Pathogenic Microorganisms in Agricultural Crops and Reduce Their Resistance. Mexico Patent Application No. MX/a/2020/012942, 2020.

24. St Thomas, C.; Guerrero-Santos, R.; D'Agosto, F. Alkoxyamine-functionalized latex nanoparticles through RAFT polymerizationinduced self-assembly in water. Polym. Chem. 2015, 6, 5405-5413. [CrossRef]

25. Armes, S.P.; Perrier, S.; Zetterlund, P.B. Introduction to polymerisation-induced self assembly. Polym. Chem. 2021, 12, 8-11. [CrossRef]

26. Yildirim, T.; Rinkenauer, A.C.; Weber, C.; Traeger, A.; Schubert, S.; Schubert, U.S. RAFT made methacrylate copolymers for reversible pH-responsive nanoparticles. J. Polym. Sci. Part A Polym. Chem. 2015, 53, 2711-2721. [CrossRef]

27. Szczepaniec, A.; Varela, K.A.; Kiani, M.; Paetzold, L.; Rush, C.M. Incidence of resistance to neonicotinoid insecticides in Bactericera cockerelli across Southwest U. S. Crop Prot. 2019, 116, 188-195. [CrossRef]

28. Cázares Alonso, N.P.; Verde Star, M.J.; López Arroyo, J.I.; Almeyda León, I.H. Evaluación de diferentes extractos vegetales contra el psílido asiático de los cítricos Diaphorina citri (Hemiptera: Liviidae). Rev. Colomb. Entomol. 2014, 40, 67-73.

29. Zhang, M.; Powell, C.A.; Zhou, L.; He, Z.; Stover, E.; Duan, Y. Chemical Compounds Effective Against the Citrus Huanglongbing Bacterium 'Candidatus Liberibacter asiaticus' In Planta. Phytopathology 2011, 101, 1097-1103. [CrossRef] [PubMed]

30. Yañez-Macias, R.; Alvarez-Moises, I.; Perevyazko, I.; Lezov, A.; Guerrero-Santos, R.; Schubert, U.S.; Guerrero-Sanchez, C. Effect of the Degree of Quaternization and Molar Mass on the Cloud Point of Poly[2-(dimethylamino)ethyl methacrylate] Aqueous Solutions: A Systematic Investigation. Macromol. Chem. Phys. 2017, 218, 1700065. [CrossRef] 
31. Mayo-Hernández, J.; Ramírez-Chávez, E.; Molina-Torres, J.; de Lourdes Guillén-Cisneros, M.; Rodríguez-Herrera, R.; HernándezCastillo, F.; Flores-Olivas, A.; Valenzuela-Soto, J.H. Effects of Bactericera cockerelli Herbivory on Volatile Emissions of Three Varieties of Solanum lycopersicum. Plants 2019, 8, 509. [CrossRef] [PubMed]

32. Livak, K.J.; Schmittgen, T.D. Analysis of Relative Gene Expression Data Using Real-Time Quantitative PCR and the $2-\Delta \Delta C T$ Method. Methods 2001, 25, 402-408. [CrossRef] [PubMed] 